Original Research Article

\title{
Evaluation of dose dependent analgesic response by extracts of Myristica fragrans on albino wistar rats: an experimental study
}

\author{
Imran Zaheer ${ }^{1 *}$, Syed Ziaur Rahman ${ }^{2}$, Rahat Ali Khan ${ }^{2}$, \\ Mehtab Parveen ${ }^{3}$, Priyamvada Sharma ${ }^{1}$
}

Department of Pharmacology,

${ }^{1}$ F.H. Medical College and Hospital, Etmadpur, Agra,

${ }^{2}$ Jawaharlal Nehru Medical College, ${ }^{3}$ Department of

Chemistry F/O Science, Aligarh

Muslim University, Aligarh,

Uttar Pradesh, India

Received: 13 July 2019

Accepted: 16 August 2019

*Correspondence to:

Dr. Imran Zaheer,

Email: imran82zaheer

@ gmail.com

Copyright: (C) the author(s), publisher and licensee Medip Academy. This is an openaccess article distributed under the terms of the Creative Commons Attribution NonCommercial License, which permits unrestricted noncommercial use, distribution, and reproduction in any medium, provided the original work is properly cited.

\begin{abstract}
Background: The objective of the study was to evaluate analgesic activity of ethanolic extract, methanol and benzene fraction of Myristica fragrans on wistar albino rats.

Methods: The present study was carried out in the department of pharmacology JNMC AMU and F.H. Medical College, Agra. The analgesic activity was evaluated by employing the Eddy's hot plate method and tail flick response method. In both the tests, Rats of either sex weighing 150-200 g were used. The total number of animals $n=36$ were allocated to six groups. Each group consist of six animals each. The response noted in animals that were tested by hot plate method was reaction time for licking/biting of both the paws before and after administration of control \& test drugs. However in Tail flick test, the pain threshold response was recorded before and after administration of control \& test drugs. The statistical analysis was done by using one-way ANOVA. The data is expressed as Mean \pm SEM. $\mathrm{P}<0.05$ was considered to be statistically significant.

Results: Ethanolic extracts and methanol fraction of $M$. fragrans showed statistically significant $(\mathrm{p}<0.001)$ increase in reaction time for licking/biting in hot plate method. On the contrary a significant increase in pain threshold was also recorded in tail flick response test. It is interesting to note that no significant degree of analgesia related to any dose of benzene fraction was observed.

Conclusions: The present study reveals the dose dependent significant analgesic activity of the extracts of $M$. fragrans i.e. ethanolic extracts and methanol fraction in both the test. However, the degree of analgesia was recorded significantly higher in groups received higher doses of extracts of $M$. fragrans.
\end{abstract}

Keywords: Analgesic activity, Myristica fragrans, Albino wistar rats

\section{INTRODUCTION}

Pain is a common subjective phenomenon which brings a patient to physician. It is associated with a number of diseases and is estimated that $80-100 \%$ of the population experience back pain at least once in the life time. ${ }^{1}$ Nonsteroidal anti-inflammatory drugs (NSAIDs) are the main stay of treatment of pain. ${ }^{2}$ It is known fact that the risk of gastrointestinal bleeding and other side effects are associated with acute and chronic use of non-steroidal anti-inflammatory drugs (NSAIDs). ${ }^{3}$ Keeping in view the gravity of adverse effects of NSAIDs it is necessary to search for new drugs with less adverse effects. In line with this many traditional medicinal plants have been used time to time for the development of new drugs with comparatively less ADRs as compare to NSAIDs. ${ }^{4}$ Recently, many natural medicines derived from medicinal plants, were considered as the effective and safer for the treatment of various diseases including inflammation and pain. ${ }^{5}$ One such medicinal plant namely Myristica fragrans is claimed for it is valuable role in reducing pain and inflammation. Hence, it was 
found worthwhile to evaluate the extracts of $M$. fragrans for their role in reducing pain in experimental animals. $M$. fragrans (Nutmeg) belongs to the family myristicaceae, is one of the important spices used in indigenous system of medicine in India. Its usefulness is reported in inflammations, cephalgia, helminthiasis, halitosis, dyspepsia, flatulence, nausea, vomiting, diarrhoea, dysentery, colic, asthma, catarrh, neuralgia, lumbago, stangury, amenorrhoea, menorrhagia, dysmenorrhoea, ulcers, liver and spleenic disorders, eye diseases, impotency, skin diseases, freckles, cracks in feet, insomnia, delirium tremens, hyperdypsia, cardiac disorders, fever and general debility. ${ }^{6}$ Earlier studies showed that ethanolic extract of $M$. fragrans have analgesic activity. ${ }^{7}$ The present study is done to validate the earlier study and to screen additionally the effect of its methanol \& benzene fraction.

\section{METHODS}

\section{Plant materials}

Seeds of $M$. fragrans were obtained from the local market. These were identified and authenticated by Chief Scientist, Raw Material Herbarium and Museum, National Institute of Science Communication and Information Resources (NISCAIR), New Delhi. A sample specimen of plant material was deposited in the NISCAIR bearing voucher number "NISCAIR/RHMD/2013/2345-125-2".

\section{Preparation of extract}

M. fragrans seeds were shade-dried. Plant material (100g) was powdered by using electrical grinder (REMI auto-mix blender, Vasai, India). The powdered plant material was extracted with $300 \mathrm{ml}$ of absolute alcohol by employing Soxhlet apparatus. The plant extract was filtered, evaporated and dry at $40^{\circ} \mathrm{C}$ on a water bath. Thus the extract material (semisolid mass) was weighed to calculate its yield in percentage. The final yield of ethanolic extract of $M$. fragrans was $17.94 \%$.

\section{Preparation of fraction}

One $\mathrm{kg}$ powder was made after crushing the dried roots of $M$. fragrans. The air-dried powdered were exhausted with $95 \%$ ethanol and the solvent was isolated by steam distillation. Under reduced pressure the extract was concentrated to dark gummy mass. The residue so obtained was fractionated by refluxing in the consecutive order with benzene, ethyl acetate and methanol. The yield of methanol and benzene fraction of $M$. fragrans was $4.17 \%$ and $5.31 \%$ respectively.

\section{Animals used}

Wistar albino rats weighing between $150-200 \mathrm{~g}$ of either sex were used. The animals were procured from the
Central Animal House and were housed in polypropylene cages bedded with husk. They were provided with standard pellet diet (Ashirwad Industries, Chandigarh) and water ad libitum. The animal room was wellventilated and maintained under standard environmental conditions throughout the experiment (temperature 18$29^{\circ} \mathrm{C}$, humidity $30-70 \%, 12$ hour light/dark cycle). Rats were acclimatized to the laboratory condition for 1 week prior to experimental use. The study followed ARRIVE guidelines and was approved by the Institutional Animal Ethics Committee.

\section{Acute toxicity study}

No toxicity of ethanolic extract of $M$. fragrans seeds was done as ethanolic extract of plant material is claimed to be safe. However, the limit toxicity of methanol and benzene fraction of $M$. fragrans in addition to acute toxicity in accordance to Organization for Economic Cooperation and Development (OECD) Guidelines 425" was done on healthy adult female rats (100-150 g). While following the limit toxicity a dose of $2000 \mathrm{mg} / \mathrm{kg}$ was administered to a group of five animals for calculation of $\mathrm{LD}_{50}$ of methanol fraction and they were observed for 14 days. However, no mortality related above limit toxicity dose was noted. ${ }^{8}$

\section{Drugs}

Pentazocine lactate (Inj. Fortwin, Ranbaxy Lab. Ltd., India).

\section{Experimental protocol}

Analgesic activity by Eddy's hot plate method

The present study was performed by using the Eddy's hot plate method (Orchid Scientifics, India). The hot plate is an electrically heated aluminium plate with a temperature ranging between $55^{\circ}$ to $56^{\circ} \mathrm{C}$.

Rats of either sex (150-200 g) were used. The response noted was reaction time of licking/biting of both paws. They were similarly screened and those responding in $<6$ sec were chosen. The paws of rats are very sensitive to heat at temperatures compared to skin.

The selected animals were placed on hot plate to record the response. The reaction time was measured at the interval of 30, 60, 90,120,150,180, 210 and 240 minutes after the administration of control and test drugs. Propylene glycol $0.3 \mathrm{ml} / 100 \mathrm{~g}$ p.o. served as control whereas Pentazocine $30 \mathrm{mg} / \mathrm{kg}$ i.p. was administered as standard drug. The cut-off time for response reaction was 30 seconds. The plate was wiped clean every time with saline if urination or defecation is found.

The test was done in all 8 groups as shown in Table 1. 
Table 1: Experimental design.

\begin{tabular}{|ll|}
\hline Groups & Medication \\
\hline $\begin{array}{l}\text { Group I (Normal } \\
\text { control) }\end{array}$ & $\begin{array}{l}\text { Propylene glycol } 0.3 \mathrm{ml} / 100 \mathrm{~g} \\
\text { p.o. }\end{array}$ \\
\hline $\begin{array}{l}\text { Group II } \\
\text { (Standard control) }\end{array}$ & Pentazocine $30 \mathrm{mg} / \mathrm{kg}$ i.p \\
\hline $\begin{array}{l}\text { Group III } \\
\text { (EEMF200) }\end{array}$ & $\begin{array}{l}\text { Ethanolic extract of } M \text {. } \text { fragrans } \\
\text { seed } 200 \mathrm{mg} / \mathrm{kg} \text { p.o. }\end{array}$ \\
\hline $\begin{array}{l}\text { Group IV } \\
\text { (EEMF400) }\end{array}$ & $\begin{array}{l}\text { Ethanolic extract of } M \text {. fragrans } \\
\text { seed } 400 \mathrm{mg} / \mathrm{kg} \text { p.o }\end{array}$ \\
\hline $\begin{array}{l}\text { Group V } \\
\text { (MFMF200) }\end{array}$ & $\begin{array}{l}\text { Methanol } \mathrm{fraction} \text { of } M \text {. fragrans } \\
\text { seed } 200 \mathrm{mg} / \mathrm{kg} \mathrm{p.o.}\end{array}$ \\
\hline $\begin{array}{l}\text { Group VI } \\
\text { (MFMF400) }\end{array}$ & $\begin{array}{l}\text { Methanol fraction of } M \text {. fragrans } \\
\text { seed } 400 \mathrm{mg} / \mathrm{kg} \text { p.o. }\end{array}$ \\
\hline $\begin{array}{l}\text { Group VII } \\
\text { (BFMF200) }\end{array}$ & $\begin{array}{l}\text { Benzene fraction of } M \text {. fragrans } \\
\text { seed } 200 \mathrm{mg} / \mathrm{kg} \text { p.o. }\end{array}$ \\
\hline $\begin{array}{l}\text { Group VIII } \\
\text { (BFMF400) }\end{array}$ & $\begin{array}{l}\text { Benzene fraction of } M \text {. fragrans } \\
\text { seed } 400 \mathrm{mg} / \mathrm{kg} \text { p.o. }\end{array}$ \\
\hline
\end{tabular}

Analgesic activity by rat tail flick test

The method is based upon the reaction of rats to heat stimulus applied to their tail. It was performed by using the analgesiometer (Orchid Scientifics, India). Rats of either sex (150-200 gm) were placed in restraining holder so that the tail between the hole and tail tip or single point $3-5 \mathrm{~cm}$ from the tip of tail are directly kept over heated nichrome wire. The time taken by the rats to withdraw the tail was recorded. Heat intensity was adjusted such that the average withdrawn latency is 3-6 sec and a maximum cut-off time of $15 \mathrm{sec}$ adopted to prevent undue tissue damage. Tail flick latency was tested at 30 min interval for 4 hours. The test was done in 8 groups as shown in Table 1.

\section{Statistical analysis}

The statistical analysis was done by using one-way ANOVA. The data is expressed as Mean \pm SEM. $\mathrm{P}<0.05$ was considered to be statistically significant.

\section{RESULTS}

Effect of ethanolic extract of $M$. fragrans seed on reaction time in rats on Eddy's hot plate

The rats in the control group responded within cut-off time of 6 seconds in all time periods. The Group II (Standard control) showed significant $(\mathrm{p}<0.001)$ increase in reaction time (seconds) as 5.48, 7.05, 8.13, 8.29, 6.96, 5.85 at interval (minutes) of 30, 60, 90, 120,150, 180 respectively. Ethanolic extracts in low (Group III/EEMF200) and high (Group IV/EEMF400 mg/kg) doses showed significant increase in reaction time (seconds) as $4.44 \quad(\mathrm{p}<0.05), 4.74 \quad(\mathrm{p}<0.001), \quad 5.33$ $(\mathrm{p}<0.001), \quad 5.60 \quad(\mathrm{p}<0.001), 4.55 \quad(\mathrm{p}<0.01)$ and 4.82

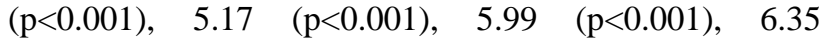
$(\mathrm{p}<0.001), 5.14(\mathrm{p}<0.001)$ at interval (minutes) of 60, 90, $120,150,180$. It was further noticed that mean response time was higher in the Group IV. The peak effect in both groups was seen at 150 mins (Table 2).

Table 2: Effect of ethanolic extract of $M$. fragrans seed on reaction time in rats on Eddy's hot plate.

\begin{tabular}{|c|c|c|c|c|c|c|c|c|c|}
\hline Groups & $0 \mathrm{~min}$ & $30 \mathrm{~min}$ & $60 \mathrm{~min}$ & $90 \mathrm{~min}$ & 120 min & $150 \mathrm{~min}$ & 180 min & 210 min & $240 \mathrm{~min}$ \\
\hline \multirow{2}{*}{$\begin{array}{l}\text { Normal } \\
\text { control }\end{array}$} & 3.89 & 3.76 & 3.89 & 3.78 & 3.95 & 3.90 & 3.89 & 3.88 & 3.85 \\
\hline & \pm 0.1 & \pm 0.14 & \pm 0.16 & \pm 0.15 & \pm 0.21 & \pm 0.1 & \pm 0.1 & \pm 0.19 & \pm 0.1 \\
\hline \multirow{2}{*}{$\begin{array}{l}\text { Positive } \\
\text { control }\end{array}$} & 3.53 & 5.48 & 7.05 & 8.13 & 8.29 & 6.96 & 5.85 & 4.24 & 3.54 \\
\hline & \pm 0.2 & $\pm 0.4 * * *$ & $\pm 0.2 * * *$ & $\pm 0.2 * * *$ & $\pm 0.3 * * *$ & $\pm 0.3 * * *$ & $\pm 0.4 * * *$ & \pm 0.46 & \pm 0.1 \\
\hline \multirow{2}{*}{ EEMF 200} & 3.74 & 3.89 & 4.44 & 4.74 & 5.33 & 5.60 & 4.55 & 3.84 & 3.74 \\
\hline & \pm 0.06 & \pm 0.05 & $\pm 0.05 *$ & $\pm 0.05 * * *$ & $\pm 0.06 * * *$ & $\pm 0.05 * * *$ & $\pm 0.06 * *$ & \pm 0.10 & \pm 0.07 \\
\hline \multirow{2}{*}{ EEMF 400} & 3.84 & 4.03 & 4.82 & 5.17 & 5.99 & 6.35 & 5.14 & 4.05 & 3.84 \\
\hline & \pm 0.07 & \pm 0.08 & $\pm 0.09 * * *$ & $\pm 0.1 * * *$ & $\pm 0.1 * * *$ & $\pm 0.1 * * *$ & $\pm 0.05 * * *$ & \pm 0.04 & \pm 0.07 \\
\hline
\end{tabular}

Reaction Time: Mean $\pm \operatorname{SEM}(\mathrm{n}=6)$ sec. ${ }^{*} \mathrm{p}<0.05$, ${ }^{* *} \mathrm{p}<0.01,{ }^{* * *} \mathrm{p}<0.001$, as compared to normal control. EEMF- Ethanolic extract of $M$. fragrans

Table 3: Effect of ethanolic extract of M. fragrans seed on reaction time in rats on tail flick test.

\begin{tabular}{|c|c|c|c|c|c|c|c|c|c|}
\hline Groups & 0 min & $30 \mathrm{~min}$ & $60 \mathrm{~min}$ & $90 \mathrm{~min}$ & $120 \mathrm{~min}$ & $150 \mathrm{~min}$ & 180 min & 210 min & $240 \mathrm{~min}$ \\
\hline $\begin{array}{l}\text { Normal } \\
\text { control }\end{array}$ & $\begin{array}{l}4.12 \\
\pm 0.01\end{array}$ & $\begin{array}{l}4.11 \\
\pm 0.0\end{array}$ & $\begin{array}{l}4.12 \\
\pm 0.0\end{array}$ & $\begin{array}{l}4.13 \\
\pm 0.0\end{array}$ & $\begin{array}{l}4.13 \\
\pm .01\end{array}$ & $\begin{array}{l}4.12 \\
\pm 0.01\end{array}$ & $\begin{array}{l}4.13 \\
\pm 0.01\end{array}$ & $\begin{array}{l}4.12 \\
\pm 0.01\end{array}$ & $\begin{array}{l}4.10 \\
\pm 0.0\end{array}$ \\
\hline $\begin{array}{l}\text { Positive } \\
\text { control }\end{array}$ & $\begin{array}{l}4.11 \\
\pm 0.02\end{array}$ & $\begin{array}{l}5.92 \\
\pm 0.3 * * *\end{array}$ & $\begin{array}{l}6.67 \\
\pm 0.1 * * *\end{array}$ & $\begin{array}{l}8.15 \\
\pm 0.1 * * *\end{array}$ & $\begin{array}{l}9.02 \\
\pm 0.34 * * *\end{array}$ & $\begin{array}{l}7.79 \\
\pm 0.20 * * *\end{array}$ & $\begin{array}{l}6.73 \\
\pm 0.18 * * *\end{array}$ & $\begin{array}{l}5.01 \\
\pm 0.10 * * *\end{array}$ & $\begin{array}{l}4.13 \\
\pm 0.02\end{array}$ \\
\hline $\begin{array}{l}\text { EEMF } \\
200\end{array}$ & $\begin{array}{l}4.06 \\
\pm 0.02\end{array}$ & $\begin{array}{l}4.89 \\
\pm 0.04\end{array}$ & $\begin{array}{l}6.02 \\
\pm 0.2 * * *\end{array}$ & $\begin{array}{l}6.79 \\
\pm 0.06 * * *\end{array}$ & $\begin{array}{l}6.39 \\
\pm 0.08 * * *\end{array}$ & $\begin{array}{l}5.26 \\
\pm 0.11 * * *\end{array}$ & $\begin{array}{l}4.52 \\
\pm 0.13\end{array}$ & $\begin{array}{l}4.09 \\
\pm 0.01\end{array}$ & $\begin{array}{l}4.04 \\
\pm 0.02\end{array}$ \\
\hline $\begin{array}{l}\text { EEMF } \\
400\end{array}$ & $\begin{array}{l}4.05 \\
\pm 0.03\end{array}$ & $\begin{array}{l}5.25 \\
\pm 0.6 * * *\end{array}$ & $\begin{array}{l}6.57 \\
\pm 0.01 * * *\end{array}$ & $\begin{array}{l}7.37 \\
\pm 0.03 * * *\end{array}$ & $\begin{array}{l}7.01 \\
\pm 0.12 * * *\end{array}$ & $\begin{array}{l}5.94 \\
\pm 0.10 * * *\end{array}$ & $\begin{array}{l}5.34 \\
\pm 0.18 * * *\end{array}$ & $\begin{array}{l}4.19 \\
\pm 0.08\end{array}$ & $\begin{array}{l}4.03 \\
\pm 0.04\end{array}$ \\
\hline
\end{tabular}

Reaction time: Mean \pm SEM $(\mathrm{n}=6)$ sec. $* \mathrm{p}<0.05, * * \mathrm{p}<0.01, * * * \mathrm{p}<0.001$, as compared to normal control. EEMF- Ethanolic extract of $M$. fragrans 
Effect of ethanolic extract of $M$. fragrans seed on reaction time in rats on tail flick test

The rats in the control group responded within cut-off time of 6 seconds in all time periods. The standard group (Group II) showed significant $(\mathrm{p}<0.001)$ increase in reaction time (seconds) as5.92, 6.67, 8.15, 9.02, 7.79, 6.73, 5.01 at interval (minutes) of 30, 60, 90, 120,150, 180, 210 respectively. Ethanolic extract in low (Group III) dose showed significant increase in reaction time (seconds) as $6.02 \quad(\mathrm{p}<0.001), 6.79 \quad(\mathrm{p}<0.001), 6.39$ $(\mathrm{p}<0.001), 5.26(\mathrm{p}<0.001)$ at the interval (minutes) of 60 , 90,120 and 150 respectively. Ethanolic extract of high (Group IV/EEMF400mg/kg) dose showed significant increase in reaction time (seconds) as $5.25(\mathrm{p}<0.001)$, $6.57(\mathrm{p}<0.001), 7.37 \quad(\mathrm{p}<0.001), 7.01 \quad(\mathrm{p}<0.001), 5.94$ $(\mathrm{p}<0.001), 5.34(\mathrm{p}<0.001)$ at interval (minutes) of 30,60 , $90,120,150$ and 180 respectively. It was further noticed that mean response time was higher in the high dose group (Group IV). The peak effect in both groups was seen at 90 mins (Table 3).

\section{Effect of methanol fraction of $M$. fragrans seed in rats on Eddy's hot plate test}

The rats in the control group responded within cut-off time of 6 seconds in all time periods. The Group II showed significant $(\mathrm{p}<0.001)$ increase in reaction time (seconds) as 5.95, 7.22, 8.39, 8.77, 7.46 and 6.41 at the interval (minutes) of 30, 60, 90, 120, 150 and 180, respectively. Methanol fraction in low (Group
V/MFMF200 $\mathrm{mg} / \mathrm{kg}$ ) and high (Group VI/MFMF400 $\mathrm{mg} / \mathrm{kg}$ ) doses showed significant increase in reaction time (seconds) as $4.66 \quad(\mathrm{p}<0.01), \quad 5.23 \quad(\mathrm{p}<0.001), \quad 5.53$ $(\mathrm{p}<0.001), 4.59 \quad(\mathrm{p}<0.05)$ and $4.79 \quad(\mathrm{p}<0.01), \quad 5.92$ $(\mathrm{p}<0.001), 6.23 \quad(\mathrm{p}<0.001), 5.00 \quad(\mathrm{p}<0.01)$ at interval (minutes) of $90,120,150,180$. It was further noticed that mean response time was higher in the large dose group (Group VI). The peak effect in both groups was seen at 150 mins (Table 4).

\section{Effect of methanol fraction of $M$. fragrans seed on tail flick test in rats}

The rats in the control group responded within cut-off time of 6 seconds in all time periods. The Group II (Standard control) showed statistically significant increase in reaction time (seconds) as $(\mathrm{p}<0.001) 5.86,6.77,8.31$, $9.13,7.81,6.72$ and $(\mathrm{p}<0.01) 4.96$ at the interval (minutes) of 30, 60, 90, 120,150, 180 and 210 respectively. Methanol fraction in low (Group V/MFMF $200 \mathrm{mg} / \mathrm{kg}$ ) dose showed significant increase in reaction time (seconds) as $4.84(\mathrm{p}<0.05), 5.90(\mathrm{p}<0.001), 6.72$ $(\mathrm{p}<0.001), 6.37(\mathrm{p}<0.001), 5.21(\mathrm{p}<0.01)$ at the interval (minutes) of $30,60,90,120$ and 150 respectively. Methanol fraction of high (Group VI/MFMF 400mg/kg) dose showed significant increase in reaction time (seconds) as $5.25 \quad(\mathrm{p}<0.001), 6.49 \quad(\mathrm{p}<0.001), \quad 7.25$ $(\mathrm{p}<0.001), \quad 7.07 \quad(\mathrm{p}<0.001), \quad 5.85 \quad(\mathrm{p}<0.001), \quad 5.23$ $(\mathrm{p}<0.001)$ at interval (minutes) of $30,60,90,120,150$ and 180 respectively. It was further noticed that mean response time was higher in the Group VI. The peak effect in both groups was seen at 90 mins (Table 5).

Table 4: Effect of methanol fraction of M. fragrans seed on reaction time in rats on Eddy's hot plate.

\begin{tabular}{|c|c|c|c|c|c|c|c|c|c|}
\hline Groups & $0 \mathrm{~min}$ & $30 \mathrm{~min}$ & $60 \mathrm{~min}$ & 90 min & $120 \mathrm{~min}$ & $150 \mathrm{~min}$ & 180 min & $210 \mathrm{~min}$ & $240 \mathrm{~min}$ \\
\hline $\begin{array}{l}\text { Normal } \\
\text { control }\end{array}$ & $\begin{array}{l}3.89 \\
\pm 0.1\end{array}$ & $\begin{array}{l}3.76 \\
\pm 0.14\end{array}$ & $\begin{array}{l}3.89 \\
\pm 0.16\end{array}$ & $\begin{array}{l}3.78 \\
\pm 0.15\end{array}$ & $\begin{array}{l}3.95 \\
\pm 0.21\end{array}$ & $\begin{array}{l}3.90 \\
\pm 0.1\end{array}$ & $\begin{array}{l}3.89 \\
\pm 0.1\end{array}$ & $\begin{array}{l}3.88 \\
\pm 0.19\end{array}$ & $\begin{array}{l}3.85 \\
\pm 0.1\end{array}$ \\
\hline $\begin{array}{l}\text { Positive } \\
\text { control }\end{array}$ & $\begin{array}{l}3.53 \\
\pm 0.2 \\
\end{array}$ & $\begin{array}{l}5.48 \\
\pm 0.4 * * *\end{array}$ & $\begin{array}{l}7.05 \\
\pm 0.2 * * *\end{array}$ & $\begin{array}{l}8.13 \\
\pm 0.2 * * *\end{array}$ & $\begin{array}{l}8.29 \\
\pm 0.3 * * *\end{array}$ & $\begin{array}{l}6.96 \\
\pm 0.3 * * *\end{array}$ & $\begin{array}{l}5.85 \\
\pm 0.4 * * *\end{array}$ & $\begin{array}{l}4.24 \\
\pm 0.46\end{array}$ & $\begin{array}{l}3.54 \\
\pm 0.1\end{array}$ \\
\hline $\begin{array}{l}\text { MFMF } \\
200\end{array}$ & $\begin{array}{l}3.46 \\
\pm 0.31\end{array}$ & $\begin{array}{l}3.84 \\
\pm 0.12\end{array}$ & $\begin{array}{l}4.31 \\
\pm 0.09\end{array}$ & $\begin{array}{l}4.66 \\
\pm 0.09 * *\end{array}$ & $\begin{array}{l}5.23 \\
\pm 0.13 * * *\end{array}$ & $\begin{array}{l}5.53 \\
\pm 0.09 * * *\end{array}$ & $\begin{array}{l}4.59 \\
\pm 0.16^{*}\end{array}$ & $\begin{array}{l}3.79 \\
\pm 0.27\end{array}$ & $\begin{array}{l}3.72 \\
\pm 0.14\end{array}$ \\
\hline $\begin{array}{l}\text { MFMF } \\
400\end{array}$ & $\begin{array}{l}3.78 \\
\pm 0.07\end{array}$ & $\begin{array}{l}3.98 \\
\pm 0.08\end{array}$ & $\begin{array}{l}4.79 \\
\pm 0.09 * *\end{array}$ & $\begin{array}{l}5.16 \\
\pm 0.23 * * *\end{array}$ & $\begin{array}{l}5.92 \\
\pm 0.19 * * *\end{array}$ & $\begin{array}{l}6.23 \\
\pm 0.13 * * *\end{array}$ & $\begin{array}{l}5.00 \\
\pm 0.24 * *\end{array}$ & $\begin{array}{l}4.01 \\
\pm 0.08\end{array}$ & $\begin{array}{l}3.77 \\
\pm 0.12\end{array}$ \\
\hline
\end{tabular}

Reaction Time: Mean \pm SEM $(\mathrm{n}=6)$ sec. $* \mathrm{P}<0.05$, $* * \mathrm{P}<0.01$, *** $<<0.001$, as compared to normal control. MFMF- Methanol fraction of M. fragrans

Table 5: Effect of methanol fraction of M. fragrans seed on reaction time in rats using tail flick test in rats.

\begin{tabular}{|c|c|c|c|c|c|c|c|c|c|}
\hline Groups & $0 \mathrm{~min}$ & $30 \mathrm{~min}$ & $60 \mathrm{~min}$ & $90 \mathrm{~min}$ & $120 \mathrm{~min}$ & $150 \mathrm{~min}$ & $180 \mathrm{~min}$ & $210 \mathrm{~min}$ & $240 \mathrm{~min}$ \\
\hline $\begin{array}{l}\text { Normal } \\
\text { control }\end{array}$ & $\begin{array}{l}4.12 \\
\pm 0.01\end{array}$ & $\begin{array}{l}4.11 \\
\pm 0.0\end{array}$ & $\begin{array}{l}4.12 \\
\pm 0.0\end{array}$ & $\begin{array}{l}4.13 \\
\pm 0.0\end{array}$ & $\begin{array}{l}4.13 \\
\pm .01\end{array}$ & $\begin{array}{l}4.12 \\
\pm 0.01\end{array}$ & $\begin{array}{l}4.13 \\
\pm 0.01\end{array}$ & $\begin{array}{l}4.12 \\
\pm 0.01\end{array}$ & $\begin{array}{l}4.10 \\
\pm 0.0\end{array}$ \\
\hline $\begin{array}{l}\text { Positive } \\
\text { control }\end{array}$ & $\begin{array}{l}4.11 \\
\pm 0.02\end{array}$ & $\begin{array}{l}5.92 \\
\pm 0.3 * * *\end{array}$ & $\begin{array}{l}6.67 \\
\pm 0.1 * * *\end{array}$ & $\begin{array}{l}8.15 \\
\pm 0.1 * * *\end{array}$ & $\begin{array}{l}9.02 \\
\pm 0.34 * * *\end{array}$ & $\begin{array}{l}7.79 \\
\pm 0.20 * * *\end{array}$ & $\begin{array}{l}6.73 \\
\pm 0.18 * * *\end{array}$ & $\begin{array}{l}5.01 \\
\pm 0.10 * * *\end{array}$ & $\begin{array}{l}4.13 \\
\pm 0.02 \\
\end{array}$ \\
\hline $\begin{array}{l}\text { MFMF } \\
200\end{array}$ & $\begin{array}{l}4.02 \\
\pm 0.06\end{array}$ & $\begin{array}{l}4.84 \\
\pm 0.11 *\end{array}$ & $\begin{array}{l}5.90 \\
\pm 0.40 * * *\end{array}$ & $\begin{array}{l}6.72 \\
\pm 0.15 * * *\end{array}$ & $\begin{array}{l}6.37 \\
\pm 0.18 * * *\end{array}$ & $\begin{array}{l}5.21 \\
\pm 0.30 * *\end{array}$ & $\begin{array}{l}4.44 \\
\pm 0.32 \\
\end{array}$ & $\begin{array}{l}4.07 \\
\pm 0.04 \\
\end{array}$ & $\begin{array}{l}4.04 \\
\pm 0.05 \\
\end{array}$ \\
\hline $\begin{array}{l}\text { MFMF } \\
400\end{array}$ & $\begin{array}{l}4.05 \\
\pm 0.06\end{array}$ & $\begin{array}{l}5.25 \\
\pm 0.1 * * *\end{array}$ & $\begin{array}{l}6.49 \\
\pm 0.40 * * *\end{array}$ & $\begin{array}{l}7.25 \\
\pm 0.13 * * *\end{array}$ & $\begin{array}{l}7.07 \\
\pm 0.25 * * *\end{array}$ & $\begin{array}{l}5.85 \\
\pm 0.28 * * *\end{array}$ & $\begin{array}{l}5.23 \\
\pm 0.47 * * *\end{array}$ & $\begin{array}{l}4.16 \\
\pm 0.19\end{array}$ & $\begin{array}{l}4.02 \\
\pm 0.09\end{array}$ \\
\hline
\end{tabular}

Reaction Time: Mean \pm SEM $(\mathrm{n}=6)$ sec. $* \mathrm{P}<0.05$, $* * \mathrm{P}<0.01, * * * \mathrm{P}<0.001$, as compared to normal control. MFMF- Methanol fraction of M. fragrans 
Table 6: Effect of Benzene fraction of M. fragrans seed of on reaction time in rats using Eddy's hot plate.

\begin{tabular}{|c|c|c|c|c|c|c|c|c|c|}
\hline Groups & 0 min & $30 \mathrm{~min}$ & $60 \mathrm{~min}$ & $90 \mathrm{~min}$ & $120 \mathrm{~min}$ & $150 \mathrm{~min}$ & $180 \mathrm{~min}$ & $210 \mathrm{~min}$ & $240 \mathrm{~min}$ \\
\hline Normal & 3.93 & 3.93 & 3.83 & 3.91 & 3.85 & 3.83 & 3.92 & 3.83 & 3.82 \\
\hline control & \pm 0.2 & \pm 0.2 & \pm 0.2 & \pm 0.2 & \pm 0.2 & \pm 0.2 & \pm 0.2 & \pm 0.2 & \pm 0.2 \\
\hline Positive & 3.84 & 5.95 & 7.22 & 8.39 & 8.77 & 7.46 & 6.41 & 4.67 & 3.86 \\
\hline control & \pm 0.0 & $\pm 0.3 * * *$ & $\pm 0.4 * * *$ & $\pm 0.3 * * *$ & $\pm 0.1 * * *$ & $\pm 0.2 * * *$ & $\pm 0.2 * * *$ & \pm 0.5 & \pm 0.07 \\
\hline BFMF & 3.87 & 3.91 & 4.08 & 4.17 & 4.21 & 4.08 & 3.87 & 3.87 & 3.75 \\
\hline 200 & \pm 0.2 & \pm 0.2 & \pm 0.2 & \pm 0.2 & \pm 0.2 & \pm 0.1 & \pm 0.2 & \pm 0.2 & \pm 0.2 \\
\hline BFMF & 3.75 & 3.79 & 3.92 & 4.26 & 4.39 & 4.20 & 3.89 & 3.74 & 3.82 \\
\hline 400 & \pm 0.2 & \pm 0.1 & \pm 0.1 & \pm 0.09 & \pm 0.1 & \pm 0.1 & \pm 0.1 & \pm 0.2 & \pm 0.1 \\
\hline
\end{tabular}

Reaction time: Mean \pm SEM $(\mathrm{n}=6)$ sec. ${ }^{*} \mathrm{p}<0.05,{ }^{*} \mathrm{p}<0.01, * * * \mathrm{p}<0.001$, as compared to normal control. BFMF- Benzene fraction of $M$. fragrans.

\section{Effect of benzene fraction of M. fragrans seed in rats on Eddy's hot plate test}

The rats in the control group responded at all intervals by 6 seconds. The rats in the control group responded within cut-off time of 6 seconds in all time periods. The standard group (Group II) of pentazocine showed significant $(\mathrm{p}<0.001)$ increase in reaction time (seconds) as 5.95, $7.22,8.39,8.77,7.46$ and 6.41 at the interval (minutes) of 30, 60, 90, 120, 150 and 180, respectively. Low dose (Group VII/BFMF200 mg/kg) and high dose (Group VIII/BFMF400mg) of Benzene fraction of $M$. fragrans seed revealed no statistically significant effects (Table 6).

\section{Effect of benzene fraction of $M$. fragrans seed on tail flick test in rats}

The rats in the control group responded within cut-off time of 6 seconds in all time periods. The Standard group (Group II) showed significant increase in reaction time (Seconds) as ( $\mathrm{p}<0.001)$ 5.86, 6.77, 8.31, 9.13, 7.81, 6.72 and $(\mathrm{p}<0.01) 4.96$ at the interval (minutes) of $30,60,90$, 120,150, 180 and 210 respectively. Low dose (Group VII/BFMF200 $\mathrm{mg} / \mathrm{kg}$ ) and high dose (Group VIII/BFMF400 mg/kg) of benzene fraction of M.fragrans seed revealed no statistically significant effects (Table 7).

Table 7: Effect of benzene fraction of Myristica fragrans seed of on reaction time in rats using tail flick test.

\begin{tabular}{|c|c|c|c|c|c|c|c|c|c|}
\hline Groups & $0 \mathrm{~min}$ & $30 \mathrm{~min}$ & $60 \mathrm{~min}$ & $90 \mathrm{~min}$ & $120 \mathrm{~min}$ & $150 \mathrm{~min}$ & $180 \mathrm{~min}$ & $210 \mathrm{~min}$ & $240 \mathrm{~min}$ \\
\hline $\begin{array}{l}\text { Normal } \\
\text { control }\end{array}$ & $\begin{array}{l}4.05 \\
\pm 0.06\end{array}$ & $\begin{array}{l}4.02 \\
\pm 0.05\end{array}$ & $\begin{array}{l}4.03 \\
\pm 0.06\end{array}$ & $\begin{array}{l}4.07 \\
\pm 0.05\end{array}$ & $\begin{array}{l}4.01 \\
\pm 0.04\end{array}$ & $\begin{array}{l}4.00 \\
\pm 0.05\end{array}$ & $\begin{array}{l}4.05 \\
\pm 0.06\end{array}$ & $\begin{array}{l}4.04 \\
\pm 0.06\end{array}$ & $\begin{array}{l}4.03 \\
\pm 0.06\end{array}$ \\
\hline $\begin{array}{l}\text { Positive } \\
\text { control }\end{array}$ & $\begin{array}{l}4.04 \\
\pm 0.04\end{array}$ & $\begin{array}{l}5.86 \\
\pm 0.05 * * *\end{array}$ & $\begin{array}{l}6.77 \\
\pm 0.1 * * *\end{array}$ & $\begin{array}{l}8.31 \\
\pm 0.2 * * *\end{array}$ & $\begin{array}{l}9.13 \\
\pm 0.26 \text { *** }\end{array}$ & $\begin{array}{l}7.81 \\
\pm 0.26 * * *\end{array}$ & $\begin{array}{l}6.72 \\
\pm 0.21 * * *\end{array}$ & $\begin{array}{l}4.96 \\
\pm 0.15 * *\end{array}$ & $\begin{array}{l}4.11 \\
\pm 0.03\end{array}$ \\
\hline $\begin{array}{l}\text { BFMF } \\
200\end{array}$ & $\begin{array}{l}4.05 \\
\pm 0.05\end{array}$ & $\begin{array}{l}4.08 \\
\pm 0.06\end{array}$ & $\begin{array}{l}4.12 \\
\pm 0.05\end{array}$ & $\begin{array}{l}4.15 \\
\pm 0.06\end{array}$ & $\begin{array}{l}4.13 \\
\pm 0.08\end{array}$ & $\begin{array}{l}4.16 \\
\pm 0.05\end{array}$ & $\begin{array}{l}4.07 \\
\pm 0.05\end{array}$ & $\begin{array}{l}4.04 \\
\pm 0.06\end{array}$ & $\begin{array}{l}4.05 \\
\pm 0.06\end{array}$ \\
\hline $\begin{array}{l}\text { BFMF } \\
400\end{array}$ & $\begin{array}{l}4.08 \\
\pm 0.05\end{array}$ & $\begin{array}{l}4.17 \\
\pm 0.04\end{array}$ & $\begin{array}{l}4.23 \\
\pm 0.04\end{array}$ & $\begin{array}{l}4.20 \\
\pm 0.05\end{array}$ & $\begin{array}{l}4.11 \\
\pm 0.04\end{array}$ & $\begin{array}{l}4.17 \\
\pm 0.05\end{array}$ & $\begin{array}{l}4.04 \\
\pm 0.04\end{array}$ & $\begin{array}{l}4.11 \\
\pm 0.05\end{array}$ & $\begin{array}{l}4.08 \\
\pm 0.05\end{array}$ \\
\hline
\end{tabular}

Reaction time: Mean \pm SEM $(n=6)$ sec. ${ }^{*} \mathrm{p}<0.05, * * \mathrm{p}<0.01, * * * \mathrm{p}<0.001$, as compared to normal control. BFMF- Benzene Fraction of Myristica fragrans.

\section{DISCUSSION}

The present study reveals the dose dependent significant analgesic activity of the extracts of $M$. fragrans in both the test.

In Eddy's hot plate and tail flick test, The Standard group of pentazocine showed significant $(p<0.001)$ increase in reaction time. Administration of ethanolic extract of M. fragrans in low $(200 \mathrm{mg} / \mathrm{kg})$ and high $(400 \mathrm{mg} / \mathrm{kg})$ doses showed statistically significant increase in reaction time. Similarly the low dose $(200 \mathrm{mg} / \mathrm{kg})$ and high dose $(400 \mathrm{mg} / \mathrm{kg})$ of methanol fraction showed significant increase in reaction time. It was also noticed that reaction time was higher in the large dose group in both ethanolic and methanol fraction. Further in ethanolic extract and methanol fraction, the peak effect in both groups was observed at 150 mins. It is interesting to note that low dose $(200 \mathrm{mg} / \mathrm{kg})$ and high dose $(400 \mathrm{mg} / \mathrm{kg})$ of Benzene fraction of $M$. fragrans seed revealed no statistically significant effects $(\mathrm{p}>0.05)$.

Phytochemical examination of $M$. fragrans showed occurrence of actual biological composite like alkaloids, steroids, flavonoids, tannins, phenolics, glycosides in essential oil. ${ }^{9}$ M. fragrans volatile oil is comprised of a mixture of terpenes and alkenylbenzene derivatives. Myristicin, safrole and elimicin constitute about $80 \%$ of the alkenylbenzene derivatives. ${ }^{10}$ Bioactive compounds including camphene, elemicin, eugenol, isoelemicin, isoeugenol, methoxyeugenol and elimicin were identified as the main constituents of $M$. fragrans seed essential oil. ${ }^{11}$. Alkaloids are commonly found to have analgesic activities. $^{12}$ The result obtained in this work demonstrated 
a high analgesic activity at low and high dose of ethanolic extract and methanol fraction of M. fragrans.

A study reported that the chemical substance extracted from Myristicaceae plants has reduced the arachidonic acid which is the precursor for prostaglandin synthesis by inhibiting the phospholipase $\mathrm{A}_{2}{ }^{13}$ Another study also reported that the antinociceptive activity of plant extracts may be due to inhibition of interleukin- $1 \beta$ and interleukin8 release by resident peritoneal cells or to suppression of prostaglandins and bradykinin. ${ }^{14}$ However, the literature supporting this direct evidence related to antinociceptive action involving either of the mechanism for inhibition of the substances is lacking.

On the contrary a study with regard to the use of scientific methodology employed for evaluation of antinociceptive effect of extracts of $M$. fragrans is in support of reporting that both the responses of hot plate test (paw licking and jumping) integrate at supraspinal structures with the $\mathrm{C}$ and $A \delta$ type I and II sensitive fibers participating in this model. ${ }^{15}$

The result of study reveals significant analgesic effect by both hot plate and tail flick tests suggesting enrichment in components (primarily non-lipid and/or aromatic compounds) that might activate a spinally-mediated analgesic pathway. However lack of various constituents in benzene fraction showed no statistically significant analgesic effects.

\section{CONCLUSION}

We conclude that, the test drug Myristica fragrans seed in its ethanolic extract and methanol fraction showed statistically significant increase in reaction time (seconds) on Eddy's hot plate and tail flick test. The study reveals the dose dependent significant analgesic activity of the extracts of Myristica fragrans seed in both the test. Thus the present drug used in traditional medicine might give a solution as an alternative remedy in pain management.

\section{Funding: No funding sources}

Conflict of interest: None declared

Ethical approval: The study followed ARRIVE guidelines and was approved by the Institutional Animal Ethics Committee (Registration No. 401/RO/C/2001/CPCSEA)

\section{REFERENCES}

1. Theophile D, Laurel NE, Benoit NT, Anatole AG, Emmanuel AA, Paul TV, et al. Antinociceptive and anti-inflammatory effects of the ethyl acetate stem bark extract of Bridelia scleroneur. Inflammopharmacology. 2006;14:42-7.

2. Burke A, Smyth EM, Fitzgerald GA. Analgesic antipyretic agents; Pharmacotherapy of gout. In: Brunton LL, Lazo JS, Parker KL, editors. Goodman and Gilman's the pharmacological basis of therapeutics. 11th ed. New York: Mmcgraw-Hill; 2006: 671-716.

3. Pilotto A, Franceschi M, Leandro G. The risk of upper gastrointestinal bleeding in elderly users of aspirin and other non-steroidal anti-inflammatory drugs: the role of gastroprotective drugs. Aging Clin. Exp. Res. 2003;15:494-49.

4. Raza M,Shaheen F, Choudhary MI, Sombati S, Rafiq A, Suria A, Atta-ur-Rahman\& De-Lorenzo RJ. Anticonvulsant activities of ethanolic extract and aqueous fraction isolated from Delphinium denudatum. J Ethnopharmacol. 2001;78:73-8.

5. Bellakhdar J, La pharmacopée Marocain Traditionnelle. Médecin Arabe Ancienne et Savoirs Populaires. Ibis Pres, Paris, 1997.

6. Phulsagar S,Dundi M, Bhagwat S, Girigaon Y. An Inside Review of Myristica fragrans Houtt. A Potential Medicinal Plant of India. 2014;1:500-13.

7. Joyce OO, Chinwe ND, Tabot PD, Bruno CC, JppKwaku. The analgesic effect of ethanolic extract of Myristica fragrans Houtt (nutmeg) on mice. Am J Pharm Tech Res. 2012;2:265-70.

8. OECD Guidelines for testing of chemicals. Acute Oral Toxicity- Up and Down Procedure. Adopted: 17th December 2001. http://www.oecd.org/chemical safety/risk-assessment/1948378.pdf. Accessed 15 July 2014.

9. Saxena R, Patil P. Phytochemical Studies on Myristica fragrance Essential Oil. Biological Forum: An Int J. 2012;4:62-4.

10. Janssen J, Lackman G. Nutmeg oil: identification and quantification of its most active constituents as inhibitors of platelet aggregation. J Ethnopharmacol. 1990;29:179-88.

11. Chirathaworn C, Kongcharoensuntorn W, Dechdoungchan T, Lowanitchapat A, Sanguanmoo P, Poovorawan Y. Myristica fragrans Houtt. methanolic extract induces apoptosis in a human leukemia cell line through SIRT1 mRNA downregulation. J Med Assoc Thai. 2007;90:2422-8.

12. Al-Shammary Hayfaa A, Malik AA, Al-Saadi S, Awatif MA. Evaluation of analgesic activity and toxicity of alkaloids in Myristica fragran s seeds in mice. J Pain Res. 2013;6:611-5.

13. Sarkar A. Herbal Toxicology. New Delhi: Discovery Publishing House Private Limited, 2009.

14. Mukherjee A, Chaliha M, Das S. Study of analgesic activity of ethanol extracts of Phlogacanthusthyrsiforus on experimental animal models. Bangladesh J Pharmacol. 2009;4:147-9.

15. Lopes L, Pereira S, Silva L, Figueiredo K, Moura B, Almeida F, Sousa F. Antinociceptive effect of topiramate in models of acute pain and diabetic neuropathy in rodents. Life Sci. 2009;84:105-10.

Cite this article as: Zaheer I, Rahman SZ, Khan RA, Parveen M, Sharma P. Evaluation of dose dependent analgesic response by extracts of Myristica fragrans on albino wistar rats: an experimental study. Int $\mathbf{J}$ Basic Clin Pharmacol 2019;8:2134-9. 